\title{
Long-Wave Infrared Photodetectors Based on
} 2D Platinum Diselenide atop Optical Cavity

\author{
Substrates
}

\section{(Supporting Information)}

Nima Sefidmooye Azar ${ }^{\dagger}$ James Bullock ${ }^{\dagger}$ Vivek Raj Shrestha ${ }^{\ddagger}$ Sivacarendran Balendhran, ${ }^{\ddagger}$ Wei Yan, ${ }^{\dagger}$ Hyungjin Kim, ${ }^{\boldsymbol{\top}, \S}$ Ali Javey, ${ }^{\boldsymbol{\top}, \S}$ and Kenneth B.

$$
\text { Crozier }{ }^{*}+, \ddagger, \|
$$

$\dagger$ Department of Electrical and Electronic Engineering, University of Melbourne, Victoria 3010, Australia

$\ddagger$ School of Physics, University of Melbourne, Victoria 3010, Australia

IDepartment of Electrical Engineering and Computer Sciences, University of California, Berkeley, CA 94720, USA

§Materials Sciences Division, Lawrence Berkeley National Laboratory, Berkeley, CA 94720, $U S A$

$\| A R C$ Centre of Excellence for Transformative Meta-Optical Systems, University of Melbourne, Victoria 3010, Australia

E-mail: kcrozier@unimelb.edu.au

This document contains 2 pages and 3 figures. 

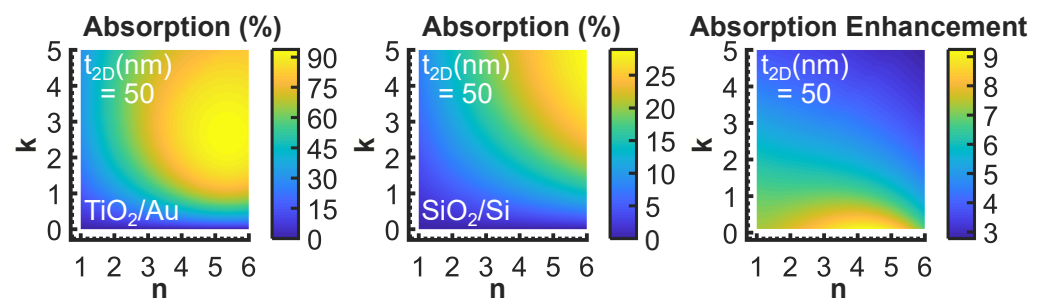

Figure S1: Optical power absorbed and absorption enhancement in the 2D material lying on the $\mathrm{TiO}_{2} / \mathrm{Au}\left(t_{s}=800 \mathrm{~nm}\right)$ and $\mathrm{SiO}_{2} / \mathrm{Si}\left(t_{s}=300 \mathrm{~nm}\right)$ substrates as functions of $n$ and $k$ with $t_{2 D}=50 \mathrm{~nm}$ at $\lambda=8.35 \mu \mathrm{m}$.

(a)

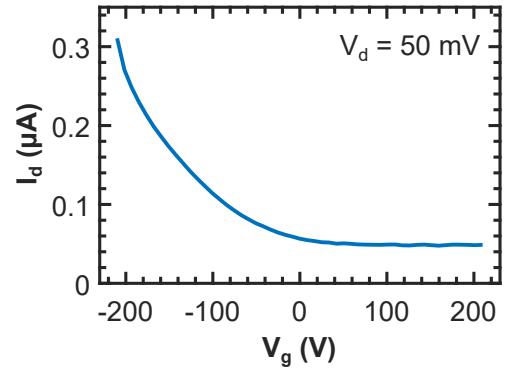

(b)

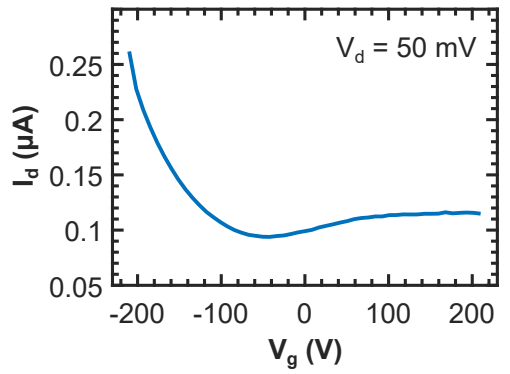

Figure S2: Drain current vs. gate voltage characteristic of few-layer $\mathrm{PtSe}_{2}$ flakes (thickness $\leq 5 \mathrm{~nm}$ ) on the $\mathrm{SiO}_{2} / \mathrm{Si}\left(t_{s}=300 \mathrm{~nm}\right)$ substrate with weak (a) p-type and (b) ambipolar behavior.

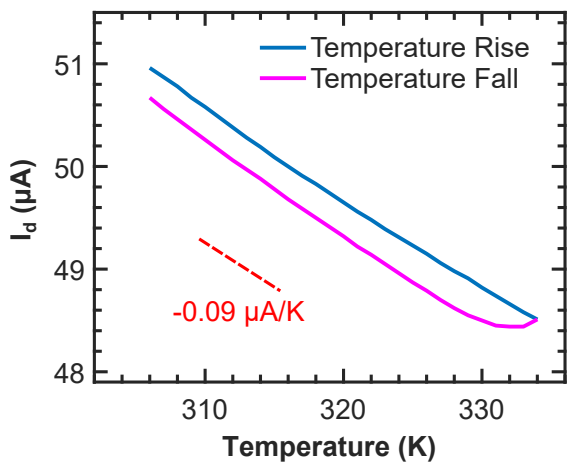

Figure S3: Drain current as a function of temperature for a $\mathrm{PtSe}_{2}$ device on the $\mathrm{SiO}_{2} / \mathrm{Si}$ $\left(t_{s}=300 \mathrm{~nm}\right)$ substrate in the dark. The drain bias was $V_{d}=50 \mathrm{mV}$, and the gate electrode was disconnected in the above measurements. 